\title{
IIR Band Pass and Band Stop Filter Design Employing Teaching-Learning based Optimization Technique
}

\author{
Damanpreet Singh \\ Sant Longowal Institute of Engineering and \\ Technology, Longowal, India
}

\author{
J.S. Dhillon \\ Sant Longowal Institute of Engineering and \\ Technology, Longowal, India
}

\begin{abstract}
In this paper newly developed teaching-learning based optimization (TLBO) algorithm is applied for designing band pass (BP) and band stop (BS) digital IIR filters. TLBO is heuristic algorithm based on the social phenomenon of teaching-learning process. The effectiveness of purposed algorithm is validated by designing the BP and BS filters by approximating the magnitude response with $L_{p}$-norm error criterion, minimizing pass band and stop band ripples along with guaranteed stability. The results obtained employing TLBO are compared to those obtained by the well known evolutionary algorithms such as hierarchical genetic algorithm, hybrid taguchi genetic algorithm and immune algorithm. The results reveal that the purposed TLBO algorithm gives better optimal filter in terms of magnitude response and ripples in pass band and stop band.
\end{abstract}

\section{Keywords}

IIR filter, teaching-learning based optimization, magnitude response, band pass, band stop, stability, $L_{p}$-approximation error.

\section{INTRODUCTION}

Digital filters are used in number of application like communication, radar signal processing, speech and image processing, biomedicine, and seismic exploration. The main purpose of using digital filter is to extract the useful information from the signal, and to remove the unwanted part of the signal. On the basis of impulse response length, digital filters are classified in to two types: finite impulse response (FIR) filter and infinite impulse response (IIR) filter [1]. IIR filter gives better performance with lesser number of coefficients than FIR for the same design specification. IIR filters are used when sharp cut off and high throughput are needed. However, there are some disadvantages of IIR digital filter such as [2]: 1) instability of IIR filter 2) multi-modal error surface. The stability problem can be easily handled by imposing stability constraints on the denominator coefficients of IIR digital filter. Due to non-linear and multimodal error surface of IIR digital filter, the classical gradient based optimization techniques cannot find the global minimum [3, 4]. In recent years, many researcher have applied number of evolutionary algorithms for the design of IIR digital filters such as: GA [5-8], immune algorithm (TIA) [9], tabu search [10], particle swarm optimization (PSO) [11, 12], Seeker optimization algorithm (SOA) [13], two-stage ensemble evolutionary algorithm [14], Gravitation search algorithm [15] and many more.

All the evolutionary and swarm intelligence based algorithms are probabilistic algorithms and require common controlling parameters like number of generations, population size, elite size, etc. Besides the common control parameters, each algorithm requires its own algorithm-specific control parameters. The performance of the above mentioned algorithms is immensely dependent upon proper tuning of the algorithm-specific parameters. The improper tuning of algorithm-specific parameters either increases the computational effort or yields the local optimal solution. To overcome the above discussed drawbacks, Rao et al. $[16,17]$ proposed a teaching-learning based optimization (TLBO) algorithm based on the natural phenomenon of teachinglearning. The implementation of TLBO does not require the determination of any algorithm specific controlling parameters which makes the algorithm robust and powerful. TLBO requires only common controlling parameters like population size and number of generations for its working.

The intent of this paper is to use the effectiveness of TLBO algorithm for designing band pass (BP) and band stop (BS) IIR filter employing $L_{p}$-norm error criterion, and pass band ripples and stop band ripples of digital IIR filter. The designed BP and BS IIR filters with TLBO algorithm are compared with hierarchical genetic algorithm (HGA) [7], hybrid taguchi genetic algorithm (HTGA) [8] and taguchi immune algorithm [TIA] [9] to find out the comparative effectiveness of the algorithm and determination of best optimal IIR filters. The paper is organized as follows: In Section 2, mathematical expression of an a IIR filter and the objective function are formulated. In Section 3, TLBO algorithm is briefly discussed for the IIR filter design problem. In Section 4, comprehensive and demonstrative sets of results and illustrations are given to make a comparison of the study with existing algorithms. Finally, Section 5 concludes the paper.

\section{FORMULATION OF IIR FILTER DESIGN PROBLEM}

IIR digital filters are characterized by the following difference equation [2]:

$$
\begin{aligned}
& y(n)=\sum_{k=0}^{\infty} h(k) x(n-k) \\
& y(n)=\sum_{k=0}^{N} b_{k} x(n-k)-\sum_{k=1}^{M} a_{k} y(n-k)
\end{aligned}
$$

where $h(k)$ is the impulse response of the filter, which is theoretically infinite in duration, $b_{k}$ and $a_{k}$ are the coefficients of the filter, $x(n)$ and $y(n)$ are the discrete input and output of the filter. The transfer function of IIR filter is defined as below:

$$
H(z)=\frac{\sum_{k=0}^{M} a_{k} z^{-k}}{1+\sum_{k=1}^{N} b_{k} z^{-k}}
$$

Where $M, N$ are numerator and denominator orders, respectively. An important part of the IIR filter design process is to find suitable values for the coefficients $b_{k}$ and $a_{k}$ such that some aspect of the filter characteristics, such as frequency response, behaves in a desired manner. An IIR digital filter 
can be expressed by the cascading first and second order sections [18] stated as:

$$
\begin{aligned}
& H(\omega, x)=A\left(\prod_{u=1}^{M} \frac{1+p_{1 u} e^{-j \omega}}{1+q_{1 u} e^{-j \omega}}\right) \\
& \times\left(\prod_{v=1}^{N} \frac{1+g_{1 v} e^{-j \omega}+g_{2 v} e^{-2 j \omega}}{1+h_{1 v} e^{-j \omega}+h_{2 v} e^{-2 j \omega}}\right)
\end{aligned}
$$

where

$$
x=\left[p_{11}, q_{11}, \ldots, p_{1 M}, q_{1 M}, g_{11}, g_{21}, h_{11}, h_{21}, \ldots, g_{1 N}, g_{2 N}, h_{1 N}, h_{2 N}, A\right]^{T}
$$
and Vector $x$ denotes the filter coefficients of dimension $S \times 1$ with $S=2 M+4 N+1$ and $A$ is the gain. The main goal of the design algorithm of digital IIR filter is to find a set of filter coefficients to minimize the magnitude approximation error function in $L_{p}$-norm $[8,9]$ and ripples in pass band and stop band.. The magnitude response is specified at $K$ equally spaced discrete frequency samples in pass-band and stopband. $E_{l}(x)$ denotes the absolute error $L_{l}$-norm of magnitude response and $E_{2}(x)$ denotes the squared error $L_{2}$-norm of magnitude response and are defined as given below:

$$
\begin{aligned}
& E_{1}(x)=\sum_{i=0}^{K}\left|H_{I}\left(\omega_{i}\right)-\right| H\left(\omega_{i}, x\right) \mid \\
& E_{2}(x)=\sum_{i=0}^{K}\left(\left|H_{I}\left(\omega_{i}\right)-\right| H\left(\omega_{i}, x\right) \mid\right)^{2}
\end{aligned}
$$

Ideal magnitude response $H_{I}\left(\omega_{i}\right)$ of IIR filter is given as:

$$
H_{I}\left(\omega_{i}\right)=\left\{\begin{array}{l}
1, \quad \text { for } \omega_{i} \in \text { passband } \\
0, \quad \text { for } \omega_{i} \in \text { stopband }
\end{array}\right.
$$

The ripple magnitudes of pass-band and stop-band are to be minimized, which are denoted by $\delta_{p}(x)$ and $\delta_{s}(x)$ respectively. Ripple magnitudes are defined as:

$$
\delta_{p}(x)=\max _{\omega_{i}}\left\{\left|H\left(\omega_{i}, x\right)\right|\right\}-\min _{\omega_{i}}\left\{\left|H\left(\omega_{i}, x\right)\right|\right\}
$$

for $_{i} \in$ passband

and

$$
\begin{array}{r}
\delta_{p}(x)=\max _{\omega_{i}}\left\{\left|H\left(\omega_{i}, x\right)\right|\right\} \\
\text { for } \omega_{i} \in \text { stopband }
\end{array}
$$

Aggregating all objectives and stability constraints, the multicriterion constrained optimization problem is stated as

Minimize $O_{1}(x)=E_{1}(x)$

Minimize $O_{2}(x)=E_{2}(x)$

Minimize $O_{3}(x)=\delta_{p}(x)$

Minimize $O_{4}(x)=\delta_{s}(x)$

Subject to: the stability constraints

$$
\begin{aligned}
& 1+q_{1 u} \geq 0(u=1,2, \ldots, M) \\
& 1-q_{1 u} \geq 0(u=1,2, \ldots, M) \\
& 1-h_{2 v} \geq 0(v=1,2, \ldots, N) \\
& 1+h_{1 v}+h_{2 v} \geq 0(v=1,2, \ldots, N) \\
& 1-h_{1 v}+h_{2 v} \geq 0(v=1,2, \ldots, N)
\end{aligned}
$$

Design of IIR filter is a multi-objective optimization problem (MOOP) as several objectives are optimized simultaneously as shown in Eq. (10a). The multiobjective constrained optimization problem for the design of digital IIR filter is converted into a scalar constrained optimization problem by using weighting method as defined below:

The objective function to be optimized is defined as:

Minimize $f(x)=\sum_{a=1}^{4} w_{a} O_{a}(x)$

Subject to: The satisfaction of stability constraints given by equation (10b) to equation (10f). where $w_{a}$ is nonnegative real number called weight. In this paper weights are taken same as given by [9].

All the poles of designed digital IIR filter should lie inside the unit circle for the filter to be stable. Therefore, the stability constraints by using the Jury method [19] have been imposed on the denominator coefficients as given by equation $(10 \mathrm{~b})$ to equation (10f). To satisfy the stability conditions coefficients have been updated with random variation as given in equation (12a) to equation (12c). The variation is given as small so that the characteristic of population in TLBO should not be changed.

$q_{1 u}=\left\{\begin{array}{cc}q_{1 u}(1-r)^{2} & ;\left(1+q_{1 u}\right)<0 \\ q_{1 u} & \text { or }\left(1-q_{1 u}\right)<0\end{array}\right.$

$h_{2 v}=\left\{\begin{array}{cc}h_{2 v}(1-r)^{2} & ;\left(1-h_{2 v}\right)<0 \\ h_{2 v} & ; \text { Otherwise }\end{array}\right.$

$h_{1 v}=\left\{\begin{array}{cc}h_{1 v}(1-r)^{2} & ;\left(1+h_{1 v}+h_{2 v}\right)<0 \\ h_{1 v} & \text { or }\left(1-h_{1 v}+h_{2 v}\right)<0 \\ & ; \text { Otherwise }\end{array}\right.$

\section{TLBO ALGORITHM FOR THE DESIGN OF IIR FILTER}

In this paper a recently developed heuristic optimization algorithm namely Teaching-learning based optimization (TLBO) proposed by Rao et al. [16, 17] is applied for the design of digital IIR filters. The useful attribute of TLBO is that it require few control parameters which remain fixed throughout the optimization process and need minimum tuning.

In the noble profession of Teaching, a teacher who is the most knowledgeable person in the class always motivates the students to acquire supreme knowledge by which they can improve their academic performances. A teacher works hard to increase the average result of the students / learners from initial level to his own level. However, in spite of the best effort of the teacher, the students not only gain knowledge on the basis of the quality of teaching delivered but also based on their own quality. There are other means in which learners can also gain knowledge by discussing and sharing ideas among other learners in form of tutorials and seminars.

In the implementation of TLBO for the design of digital IIR filter the population is analogous to $L$ number of learners in a class and each learner is assigned $S$ subjects (filter coefficients). The $i^{\text {th }}$ learner is represented as 
$X_{i}=\left[x_{i 1}, x_{i 2}, \ldots . x_{i S}\right]$ and $f\left(X_{i}\right)$ represent the fitness function for $i^{\text {th }}$ learner. The functioning of TLBO based upon two main phases namely Teacher phase and Learner phase.

\section{- Teacher Phase}

A learner having the minimum value of fitness function value calculated using Eq. (9) is designated as teacher $x t_{j}^{t}$ for current iteration $t$. A teacher tries to increase / improve the mean score of all the learners in each of the subject allotted towards its own mean score. So, the mean fitness of the class is increased by the teacher according to his / her own capability. The mean $\left(\tau_{j}\right)$ for $S$ subjects allotted to the students is evaluated and a randomly weighted differential vector $\left(\right.$ Diff $\left.f_{j}\right)$ from current mean and various desired mean vectors [20] is calculated as shown below:

$$
\begin{aligned}
& \tau_{j}^{t}=\frac{1}{L} \sum_{i=1}^{L}\left(x_{i, j}^{t}\right) \quad(j=1,2, \ldots, S) \\
& \text { Diff }_{j}^{t}=\left(x t_{j}^{t}-\left(T F \times \tau_{j}^{t}\right) \times R() \quad(j=1,2, \ldots, S)\right.
\end{aligned}
$$

where

$\tau_{j}^{t}$ is mean of $j^{\text {th }}$ subject for all learners of a class; $x t_{j}^{t}$ is the score of the teacher in $j^{\text {th }}$ subject; $T F$ is the teaching factor; $R$ is a uniform generated random number between $(0,1)$.

The teaching factor $(T F)$ is one of the vital aspect that facilitates the convergence of TLBO. The value of $T_{f}$ decides about the volume of effect a teacher has on the output of a learner. In this paper the value of $T F$ is heuristically selected as 1 or 2 as shown below:

$$
T F=R O U N D(1.0+R())
$$

The weighted differential vector $\left(\right.$ Diff $\left._{j}\right)$ generated using Eq. (14) is added to current score of learners in different subjects to generate new learners:

$$
x n e w_{i j}^{t}=x_{i j}^{t}+\text { Diff }_{j}^{t} \quad(j=1,2, \ldots, S)
$$

The newly generated learner with a better fitness value replaces the existing learner in the class.

\section{- Learner Phase}

The concept of tutorials and seminars of class room teaching system is followed in the learner phase as the knowledge acquired by the learners / students in teacher phase is further disseminated among learners themselves through discussions, sharing of notes and presentations. Two target learners namely $i$ and $m$ are selected randomly such that $i \neq m$. The resultant new learners after sharing / exchange of know-how are generated as follows:

$$
\text { xnew }_{i j}^{t}=\left\{\begin{array}{l}
x_{i j}^{t}+R() \times\left(x_{i j}^{t}-x_{m j}^{t}\right) ; f\left(X_{i}\right)<f\left(X_{m}\right) \\
x_{i j}^{t}+R() \times\left(x_{m j}^{t}-x_{i j}^{t}\right) ; \text { Otherwise }
\end{array}\right\}
$$

where

$(j=1,2, \ldots, S)$

After one successful completion of Teacher and Learner phase, algorithm is made to update teacher value before the start of next iteration. In this TLBO algorithm maximum number of iterations is chosen as the stopping criterion. If the stopping criterion is not satisfied, the above procedure is repeated with incremented $t$ value

\section{DESIGN EXAMPLES AND COMPARISONS}

For the purpose of comparison design conditions taken for designing BP and BS filters are same as [7] and are given in Table 1 . The frequency range from 0 to $\pi$ is divided in to 200 frequency sampling points. In the proposed TLBO approach the combination of four criteria, $L_{l}$-norm approximation error, $L_{2}$-norm approximation error, ripple magnitudes of pass-band and ripple magnitude of stop-band are considered for designing IIR filter. These four criteria are contrary to each other in most situations. The filter designer needs to adjust the weights of criteria to design the filter depending on the filter specifications. For the purpose of comparison the weights $w_{l}$, $w_{2}, w_{3}$ and $w_{4}$ are set same as in [9] for BP and BS filters respectively. The performance of filter designed with TLBO algorithm are presented and compared with the results obtained by [7], [8] and [9] in Table 2 and Table 3 for BP and BS filters respectively. The obtained magnitude response of BP and BS filters designed with TLBO and [7], [8] and [9] are presented in Figure 1 and Figure 2 for the purpose of comparison. The best optimized numerator coefficients and denominator coefficients obtained by the TLBO approach for BP and BS filters are given by Eq. (18) and Eq. (19) respectively. The obtained results reveal that the purposed approach gives the better performance in terms of magnitude response and ripples in pass band and stop band.

$$
\begin{gathered}
H_{B P}(z)=0.02807718\left(\frac{\left(z^{2}-0.1788042 z-0.9907626\right)\left(z^{2}+0.152085 z-0.8536902\right)}{\left(z^{2}-0.6290708 z+0.7749901\right)\left(z^{2}+0.0057888 z+0.5494546\right)}\right) \times \\
\left(\frac{\left(z^{2}-0.08027664 z-0.9487353\right)}{\left(z^{2}+0.6330091 z+0.7733375\right)}\right) \\
H_{B S}(z)=0.4394692 \frac{\left(z^{2}+0.4366674+1.000583\right)\left(z^{2}-0.4367452+1.000652\right)}{\left(z^{2}-0.815700 q+0.5407802\right)\left(z^{2}+0.8156245+0.540886\right)}
\end{gathered}
$$

Table 1. Prescribed design conditions for BP and BS filters

\begin{tabular}{|c|c|c|c|}
\hline Filter type & Pass band & Stop band & Order \\
\hline BP & $0.4 \pi \leq \omega \leq 0.6 \pi$ & $0 \leq \omega \leq 0.25 \pi$ and $0.75 \leq \omega \leq \pi$ & 6 \\
\hline BS & $0 \leq \omega \leq 0.25 \pi$ and $0.75 \leq \omega \leq \pi$ & $0.4 \pi \leq \omega \leq 0.6 \pi$ & 4 \\
\hline
\end{tabular}


Table 2. Design results for BP filter

\begin{tabular}{|c|c|c|c|c|c|c|}
\hline Method & $L_{1}$-norm error & $\mathbf{L}_{2}$-norm error & \multicolumn{3}{|c|}{$\begin{array}{l}\text { Pass-band performance } \\
\text { (Ripple magnitude) }\end{array}$} & $\begin{array}{l}\text { Stop-band performance } \\
\text { (Ripple magnitude) }\end{array}$ \\
\hline TLBO Approach & 1.2762 & 0.1789 & $0.9886 \leq$ & $\begin{array}{l}\mid H\left(e^{j \omega}\right) \\
(0.0171)\end{array}$ & $\leq 1.005$ & $\begin{array}{c}\left|H\left(e^{j \omega}\right)\right| \leq 0.525 \\
(0.0525)\end{array}$ \\
\hline TIA Approach [9] & 1.6119 & 0.2191 & $0.9806 \leq$ & $\begin{array}{l}\mid H\left(e^{j \omega}\right) \\
(0.0194)\end{array}$ & $\leq 1.000$ & $\begin{array}{c}\left|H\left(e^{j \omega}\right)\right| \leq 0.0658 \\
(0.0658)\end{array}$ \\
\hline HTGA Approach [8] & 1.9418 & 0.2350 & $0.9760 \leq$ & $\begin{array}{l}\mid H\left(e^{j \omega}\right) \\
(0.0234)\end{array}$ & $\leq 1.000$ & $\begin{array}{c}\left|H\left(e^{j \omega}\right)\right| \leq 0.0711 \\
(0.0711)\end{array}$ \\
\hline TIA Approach [7] & 5.2165 & 0.6949 & $0.8956 \leq$ & $\begin{array}{l}\mid H\left(e^{j \omega}\right) \\
(0.1044)\end{array}$ & $\leq 1.000$ & $\begin{array}{c}\left|H\left(e^{j \omega}\right)\right| \leq 0.1772 \\
(0.1772)\end{array}$ \\
\hline
\end{tabular}

Table 3. Design results for BS filter

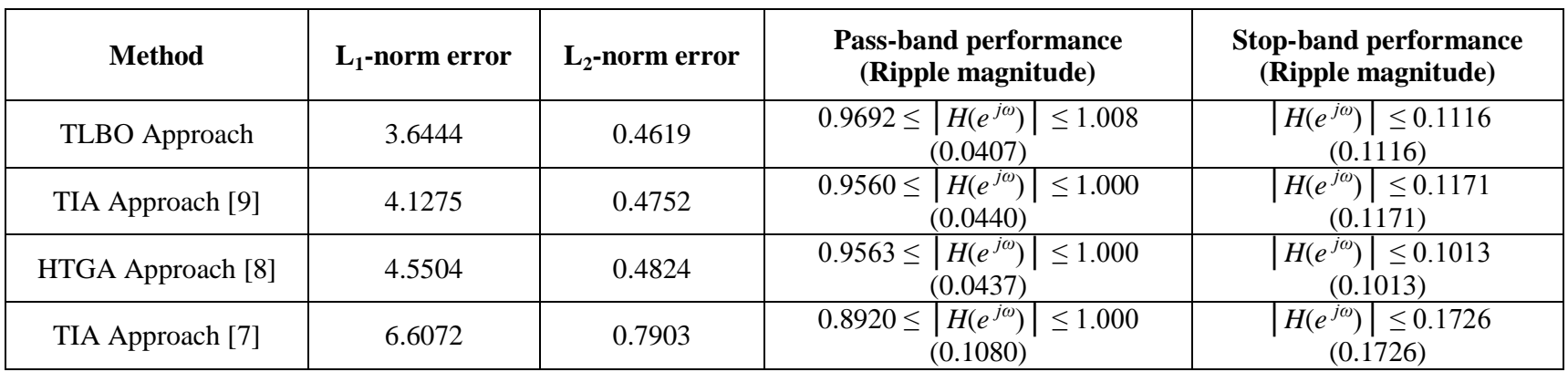
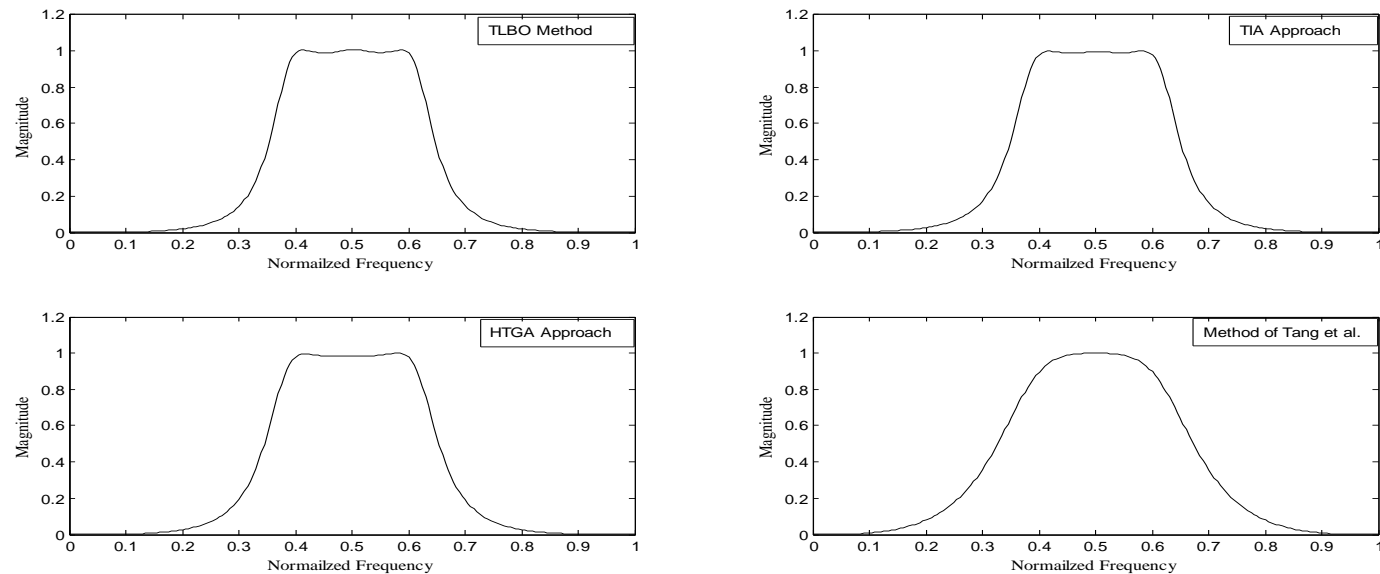

Fig 1: Magnitude responses of BP filter using TLBO approach and the method given in [9], [8] and [7]
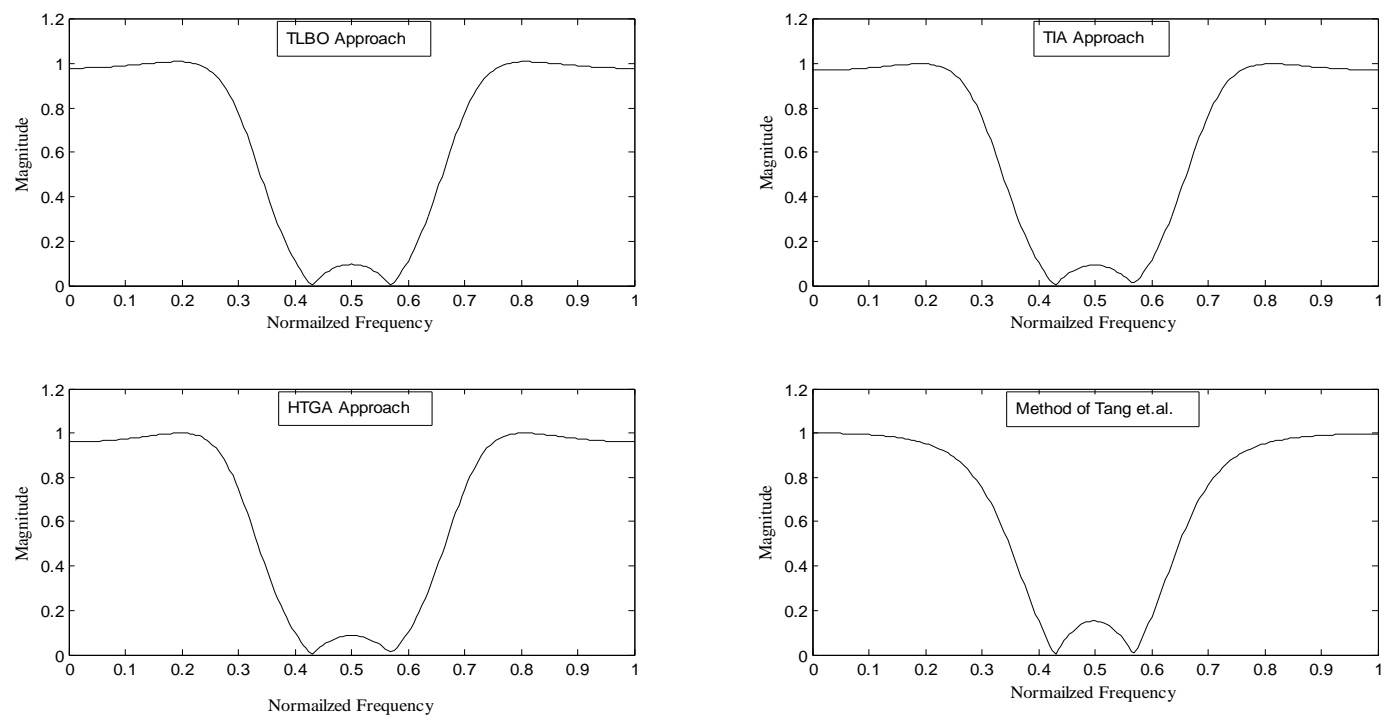

Fig 2: Magnitude responses of BS filter using TLBO approach and the method given in [9], [8] and [7] 


\section{CONCLUSION}

A heuristic optimization algorithm namely TLBO is successfully applied to design BP and BS digital IIR filter. The designed optimal filters obtained by employing TLBO meet the stability criterion and gives better performances in terms of $L_{p}$-approximation error for magnitude response and ripples in pass band and stop band in comparison to GA based methods. The main characteristics of the TLBO algorithm over other GA methods are its simplified numerical structure and its independence on a number of parameters to define the algorithm's performance. TLBO is a powerful search and applicable optimization method for the problem of digital filter design problems.

\section{REFERENCES}

[1] J. G. Proakis and D. G. Manolakis, Digital Signal Processing: Principles, Algorithms, and Applications. New Delhi: Pearson Education, Inc., 2007.

[2] A. V. Oppenheim, et al., Discrete-Time Signal Processing. NJ, Englewood Cliffs: Prentice Hall, 1999.

[3] A. Antoniou, Digital Signal Processing: Signals, Systems and Filters: McGraw Hill, 2005.

[4] W.-S. Lu and A. Antoniou, "Design of digital filters and filter banks by optimization: a state of the art review," presented at the Proceeding of European Signal Processing Conference, Finland, 2000.

[5] S. P. Harris and E. C. Ifeachor, "Automatic design of frequency sampling filters by hybrid genetic algorithm techniques," IEEE Transactions on Signal Processing, vol. 46, pp. 3304-3314, 1998

[6] J. H. Li and F. L. Yin, "Genetic optimization algorithm for designing IIR digital filters," Journal of China Institute of Communications China, vol. 17, pp. 1-7, 1996.

[7] K. S. Tang, et al., "Design and optimization of IIR filter structure using hierarchical genetic algorithms," IEEE Transactions on Industrial Elecronics, vol. 45, pp. 481487, 1998.

[8] J.-T. Tsai, et al., "Optimal design of digital IIR filters by using hybrid taguchi genetic algorithm," IEEE Transactions on Industrial Electronics, vol. 53, pp. 867879, 2006.

[9] J.-T. Tsai and J.-H. Chou, "Optimal design of digital IIR filters by using an improved immune algorithm," IEEE Transactions on Signal Processing, vol. 54 pp. 45824596, 2006
[10] A. Kalinli and D. Karaboga, " A new method for adaptive IIR filter design based on Tabu search algorithm," International Journal of Electronics and Communications (AË̈), vol. 59, pp. 111-117, 2005.

[11] S. Chen and B. L. Luk, "Digital IIR filter design using particle swarm optimisation," International Journal of Modelling, Identification and Control, vol. 9, pp. 327335, 2010.

[12] P. Upadhyay, et al., "Craziness based particle swarm optimization algorithm for IIR system identification problem," AEU - International Journal of Electronics and Communications, vol. 68, pp. 369-378, 2014.

[13] C. Dai, et al., "Seeker optimization algorithm for digital IIR filter design," IEEE Transactions on Industrial Electronics, vol. 57, pp. 1710-1718, 2010.

[14] B. Li, et al., "Fixed-point digital IIR filter design using two-stage ensemble evolutionary algorithm," Applied Soft Computing vol. 13, pp. 329-338, 2013.

[15] S. K. Saha, et al., "Gravitation search algorithm: Application to the optimal IIR filter design," Journal of King Saud University-Engineering Sciences vol. 26, pp. 69-81, 2014.

[16] R. V. Rao, et al., "Teaching-learning-based optimization: a novel method for constrained mechanical design optimization problems," Computer-Aided Design, vol. 43, pp. 303-315, 2011.

[17] R. V. Rao, et al., "Teaching-learning-based optimization: a novel optimization method for continuous non-linear large scale problems," Information Sciences, vol. 183, pp. 1-15, 2012.

[18] G. Vanuytsel, et al., "Efficient hybrid optimization of fixed-point cascaded IIR filter coefficients," in IEEE International Conference on Instrumentation and Measurement Technology, Anchorage, AK, 2002, pp. 793-797.

[19] I. Jury, Theory and Application of the Z-Transform Method New York: Wiley, 1964.

[20] M. Singh, et al., "Optimal coordination of directional over-current relays using Teaching Learning-Based Optimization (TLBO) algorithm," International Journal of Electrical Power and Energy Systems vol. 50, pp. 3341, 2013. 